\title{
ESTIMATION AND UTILIZATION OF STRUCTURE ANISOTROPY IN FORMING PIECES
}

\author{
MAROS MARTINKOVIC \\ Slovak University of Technology in Bratislava Faculty of Materials Science and Technology in Trnava, \\ J. Bottu 25, 91724 Trnava, Slovak Republic \\ ${ }^{\#}$ E-mail: maros.martinkovic@stuba.sk
}

Submitted September 2, 2016; accepted December 7, 2016

\begin{abstract}
Keywords: Stereology, Grain boundary, Fibres, Orientation, Deformation
In most of cases, technological processing leads to microstructure anisotropy of material. Results obtained from the analysis of anisotropy can be used for evaluation of material properties and parameters of technology processes. Stereological metallography was used for describing of structure anisotropy. In case of plastically deformed material, degree of orientation of grain boundaries was determined. Grain boundaries are decomposed into isotropic, planar and linear oriented components - specific surface area of grain boundaries and these parameters were measured using oriented test lines stereology method. These results were used for estimation of local plastic deformation using conversion model of orientation grain boundaries to deformation of grains. Our proposed conversion method enables estimation of local plastic deformation in arbitrary place of body with arbitrary state of initial deformation. In case of short fibre reinforced thermoplastics oriented test plane stereology method was used for estimation of experimental orientation of fibres. Degree of orientation was estimated from the number of intersections of fibres with test planes and from these results components of an orientation tensor are estimated. This method is very simple in comparison with a current method which is based on measurement of dimensions and orientation of lot of fibre intersections in analyzed plane.
\end{abstract}

\section{INTRODUCTION}

Anisotropy of microstructure in case of forming of metal or plastics depends on technology parameters of processes. In case of deformation of metals grain boundaries orientation can be observed, in case of short fibre reinforced plastics orientation of fibres.

In the polycrystalline material (metal, alloy) the main microstructural parameter is grain boundary surface interface between individual grains. In case of isotropic non deformed structure the grains have isometric dimension mean grain size or specific surface area of grain boundaries can be measured. In case of anisotropic plastically deformed structure the grains have anisometric dimension, it is necessary to describe their anisotropy [1]. The anisotropic microstructure is decomposed into isotropic, planar and/or linear oriented components - specific surface area of grain boundaries and these parameters are measured using stereology [2]. Degree of grain boundary orientation is estimated as ratio of oriented specific surface area to total specific surface area. These results can be used for estimation of local plastic deformation in arbitrary places in volume of forming pieces. Real state of grain shape is quit impossible to describe, therefore model of conversion of degree of grain boundary orientation to deformation based on an idealized shape (tetrakaidecahedron) of grains has been proposed.
Fibre orientation in short fibre reinforced thermoplastics depends on injection moulding parameters. The orientation of simple fibre may be defined by the two angles $\theta$ and $\Phi$. In a short fibre reinforced thermoplastic component there are frequently millions of fibres, therefore each individual fibre orientation specifying is very impractical. The fibres orientation in space can be described by the probability distribution function, $\Psi(\theta, \Phi)$. Nevertheless the measurement is relatively complicated. Simpler stereology method can be also used for estimation of experimental orientation of fibres. Degree of orientation is estimated as oriented specific length of fibres (specific length of linear oriented components) to total specific length of fibres (specific length of all components). These results of measurement cannot be directly compared with components of orientation tensor, so the method of estimation of main three components of the orientation tensor must be developed.

\section{ANISOTROPY OF DEFORMED MATERIAL}

In the polycrystalline material the main microstructural parameter with significant dependence on deformation is grain boundary - i.e. surface interface between individual grains. In case of anisotropic structure the grains have anisometric dimensions - are oriented. 
There are a few ways how to measure grain orientation, for instance estimation of microstructural anisotropy tensor [6]. Another way is scalar measurement of anisotropy - i.e. determination of degree of orientation. The anisotropic microstructure is decomposed into isotropic, planar and linear oriented components using stereology methods [1] and the both the oriented part of specific surface area $\left(S_{V}\right)_{O R}$ and the total specific surface area $\left(S_{V}\right)_{\text {TOT }}$ of grain boundary are estimated. Degree of grain boundary orientation $O$ is estimated as $\left(S_{V}\right)_{O R}$ to $\left(S_{V}\right)_{\text {TOT }}$ ratio. Grain boundary orientation caused by deformation $\varphi$ is the same as direction of deformation, as it is seen in Figure 1 (a - undeformed anisotropic structure, b, c, d - anisotropic structure due to various types of deformation). $\varphi$ is true (logarithmic) strain defined from dimensions of elementary body before deformation $l_{0}$ and after deformation 1 as

$$
\varphi=\int_{l_{0}}^{l} \frac{1}{l_{0}} d l
$$

On a metallographic cut test lines were placed perpendicular and parallel to the grain boundaries orientation effected by straining (see Figure 1). Isotropic,

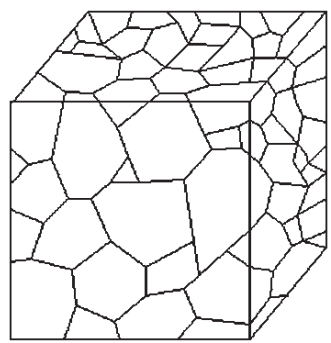

a)

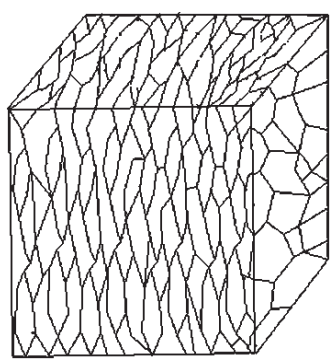

c)

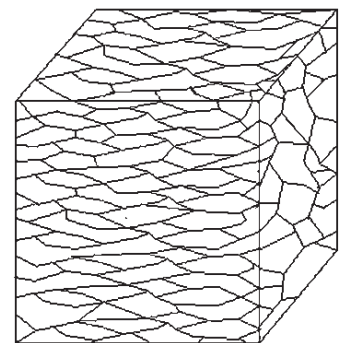

b)

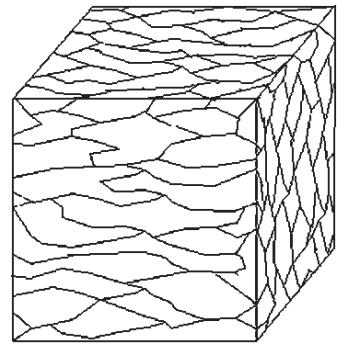

d)
Figure 1. Isometric no deformed structure (a) and anisotropic structure due to various types of deformation (b, c, d).

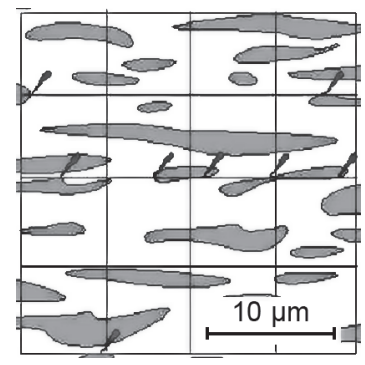

a)

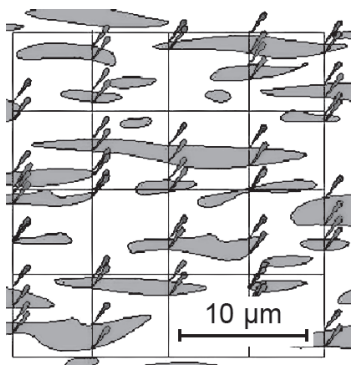

b)
Figure 2. Principle of oriented test lines stereology method. planar and linear oriented specific surface areas of grains are estimated from the specific number (number to unit of length) of parallel test lines intersections with grain boundaries and perpendicular lines ones (see Figure 2) according to [1]. But orientation is not the same as deformation; therefore it is necessary to develop a model of conversion of grain boundary orientation to deformation.

\section{Local strain estimation}

It is completely impossible to describe actual shape of the grain in material structure exactly. Therefore deformation of various idealized grain shapes can be investigated. For instance crystals can be modeled by regular polyhedron - tetrakaidecahedron [2]. One method is based on dependence of the ratio of relative surface area of grain boundaries in deformed state $S_{V}$ and undeformed state $S_{V 0}$ to strain [3]. The method requires knowledge of the parameter of structure in case of zero value of initial deformation, which is unknown in most of cases and this parameter is not the same in the whole volume of pieces and it depends on grain size.

Our conversion method was based on analysis of orientation - deformation relation of a grain. Dependence of true strain $\varphi$ on orientation $O$ was derived from three basic equations - definition of deformation (Equation 1), definition of degree of orientation and invariability of volume ( $V_{0}=V$ - initial volume is equal volume after plastic deformation). The solution of the system includes one free parameter - grain size. Solution of the system of equations for used idealised grain shapes is independent on the initial dimension of grain - strain depends only on shape of grain and it does not depend on its dimension. Due to it the method enables estimation of local plastic deformation from estimation of microstructure anisotropy in arbitrary place of body with arbitrary state of initial deformation. The solution and the result are relative complicated, so detailed

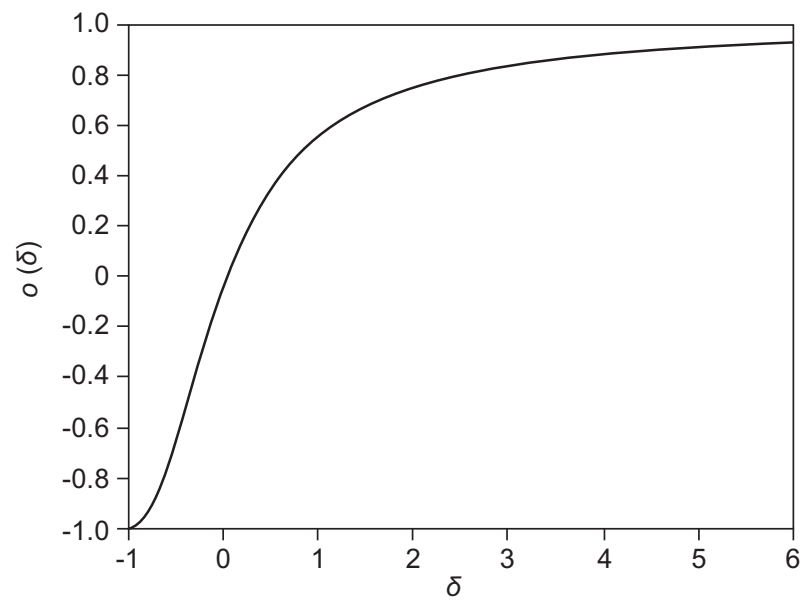

Figure 3. Dependence of orientation on deformation. 
description is in [5]. Correlation between orientation and deformation is illustrated in Figure 3, where relationship between strain $\delta$ and true strain $\varphi$ is

$$
\varphi=\ln (1+\delta)
$$

Analysis of technology process of cold drawing was realized. The semi-product for wire was hot rolled wire from ASTM 1006 steel. This hot rolled wire was cold drawn in two steps with increasing of diameter reduction and simultaneous decreasing of length and the final second step was analyzed. The diameter of semiproduct was $5.5 \mathrm{~mm}$, diameter of the wire after first drawing was $4.7 \mathrm{~mm}$ and diameter of the wire after second drawing was $3.99 \mathrm{~mm}$. Scheme of longitudinal

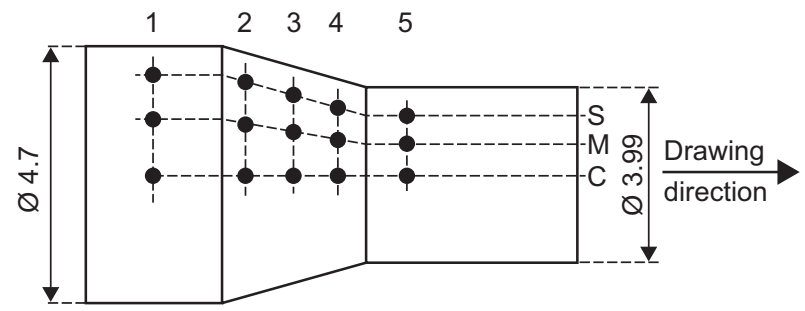

Figure 4. Scheme of longitudinal section of the sample with places for the analysis.

Table 1. Grain boundaries orientation $O$ on each analysed places and corresponding deformation $\varphi$.

\begin{tabular}{cccccc}
\hline & \multicolumn{5}{c}{$O$} \\
\cline { 2 - 6 } Place & 1 & 2 & 3 & 4 & 5 \\
\hline S & 0.32 & 0.33 & 0.37 & 0.40 & 0.48 \\
M & 0.39 & 0.41 & 0.42 & 0.50 & 0.52 \\
C & 0.35 & 0.36 & 0.39 & 0.49 & 0.51 \\
\hline \hline & \multicolumn{7}{c}{$\varphi$} \\
Place & 1 & 2 & 3 & 4 & 5 \\
\hline S & 0.316 & 0.326 & 0.371 & 0.405 & 0.504 \\
M & 0.394 & 0.417 & 0.429 & 0.530 & 0.558 \\
C & 0.348 & 0.359 & 0.394 & 0.517 & 0.544 \\
\hline
\end{tabular}

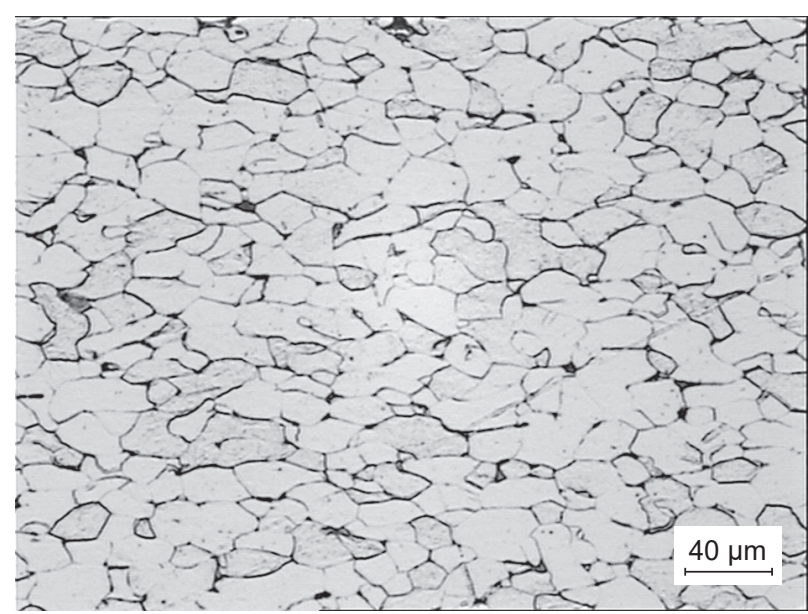

Figure 5. Structure of wire in front of drawing die (place M 1). section of the sample with fifteen places for the analysis is in Figure 4. Example of microstructures of wire in front of drawing die in place M1 is in Figure 5, structure of wire behind the drawing die in place S5 is in Figure 6. Measured and calculated degree of grain boundaries orientation in each analyzed places and corresponding deformation $\varphi$ converted according the method are in Table 1. Orientation and deformation is not equal in cross section of wire. The smallest is near the surface of wire, the greatest in the middle of wire radius in all places along the wire. It means that due to deformation strengthening during drawing wire deformation makes heavy influences on the materials properties in the middle of wire radius.

\section{Anisotropy of injection molded parts}

The orientation of one fibre can be defined by the two angles: angle $\theta$ and angle $\Phi$ shown in Figure 7 . The components of $p_{i}$ are described with the angles $\theta$ and $\Phi$, as follows:

$$
p_{1}=\sin \theta \cdot \cos \Phi, p_{2}=\sin \theta \cdot \sin \Phi, p_{3}=\cos \theta
$$

where

$$
\theta=\cos ^{-1}\left(\frac{\mathrm{b}}{\mathrm{a}}\right)
$$

The components of fibre orientation tensor for a group of $n$ fibres are calculated as follows:

$a_{i j}=\frac{1}{n}\left(\sum_{k=1}^{n} p_{i}^{k} p_{j}^{k}\right)=\left(\begin{array}{lll}a_{x x} & a_{x y} & a_{x z} \\ a_{y x} & a_{y y} & a_{y z} \\ a_{z x} & a_{z y} & a_{z z}\end{array}\right) i, j=x, y, z$

Three main independent components for an individual fibre are as follows:

$$
a_{x x}=\left(1-\frac{b^{2}}{a^{2}}\right) \cos ^{2} \Phi, a_{y y}=\left(1-\frac{b^{2}}{a^{2}}\right) \sin ^{2} \Phi, a_{z z}=\frac{b^{2}}{a^{2}}
$$

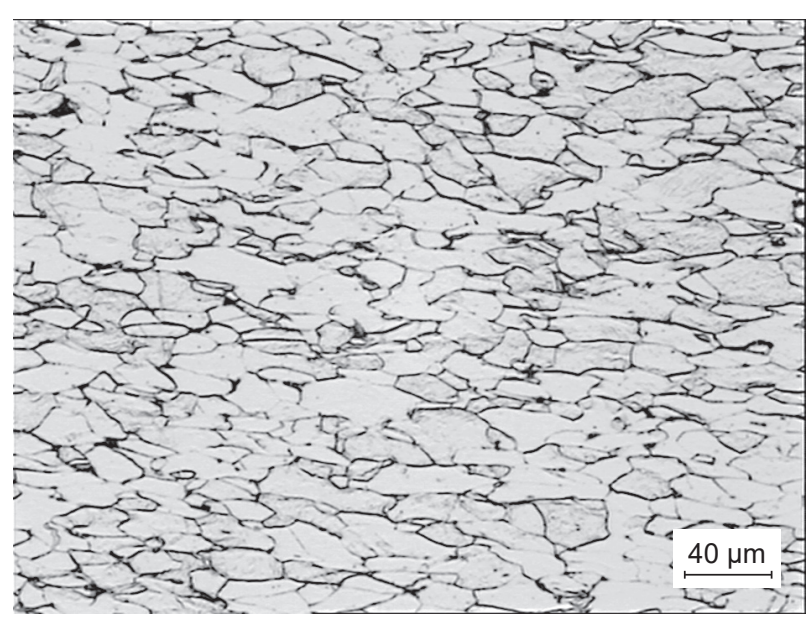

Figure 6. Structure of wire behind the drawing die (place S5). 
Orientation tensor is relative simple estimated from the measurement of dimensions and angles of a lot of fibre intersections with analysed plane, but the measurement is more complicated. The fibre must not be any size; they are perfectly circular in cross-section and have the same mean diameter.

Stereological metallography can be also used for estimation of experimental orientation of fibres [1]. In the case of short glass fibres reinforced thermoplastic its structure consists of thermoplastic matrix and reinforcing fibres, which has some preferred orientation in most of cases - the structure is anisotropic. Determination of orientation degree is the way of scalar measurement of structure anisotropy. The anisotropic microstructure is decomposed into isotropic, planar or linear oriented components using stereology methods and the both the oriented parts of specific length of linear elements in space $\left(L_{V}\right)_{O R}$ and the total specific length $\left(L_{V}\right)_{T O T}$ of fibres are estimated. Degree of fibre orientation $O$ is estimated as $\left(L_{V}\right)_{O R}$ to $\left(L_{V}\right)_{T O T}$ ratio. Oriented test plane method is used [1]. Test planes are placed perpendicular and parallel to the orientation direction (see Figure 9). Isotropic, planar and linear oriented specific length of fibres are estimated from the specific number $P_{A}$ (number per unit test area) of cross-sections between test perpendicular plane and fibres according to [1]. The fibres can be any size and any
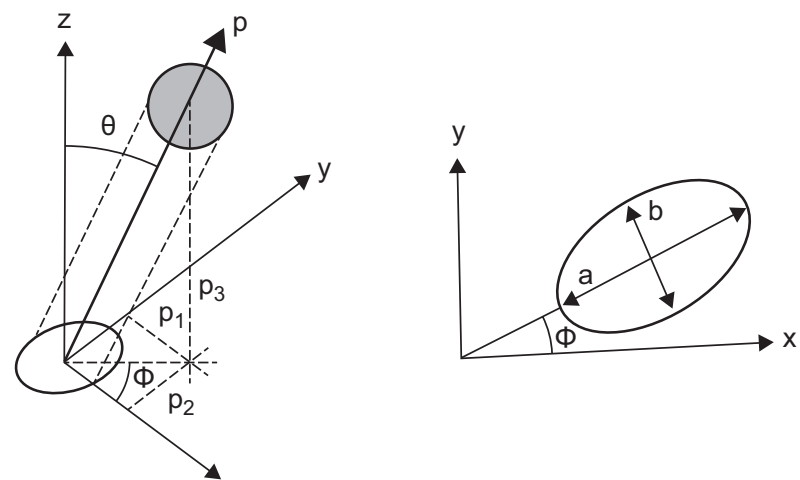

Figure 7. Orientation of a single fibre (in space and in section plane) in polar coordinates by the two angles $(\theta, \Phi)$.

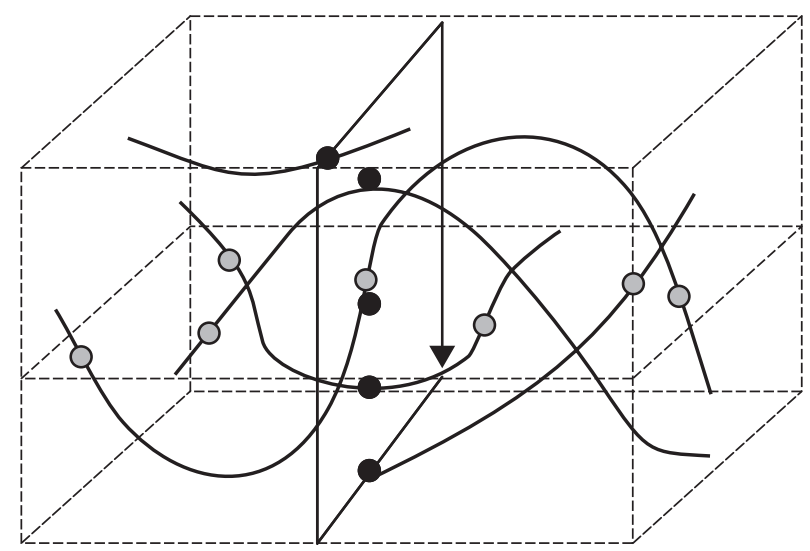

Figure 8. Principle of oriented test planes stereology method.

shape. These results of measurement cannot be directly compared with components of orientation tensor, so the method of estimation of main three components of the orientation tensor has been proposed. The main three components of the second-order orientation tensor using stereology can be calculated as follows:

$$
a_{i i}=\frac{P_{A i}}{P_{A x}+P_{A y}+P_{A z}}
$$

where $i=x, y, z ; P_{A}$ is number of cross-sections between test plane and fibres [-]; $A_{i}$ is investigated area $\left[\mathrm{mm}^{2}\right]$; $P_{A i}$ is number of cross-sections between test plane (TP) and fibres (see Figure 9) per unit of the test area $\left[\mathrm{mm}^{-2}\right]$

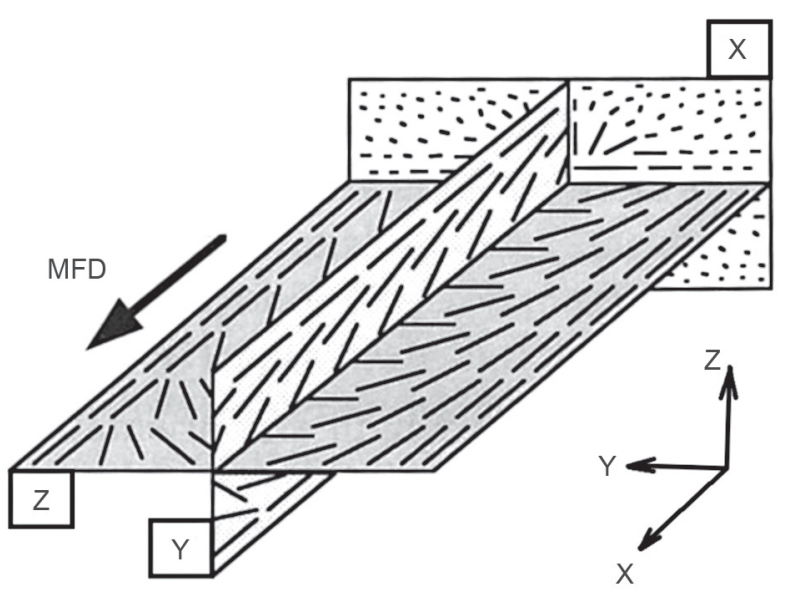

Figure 9. Test planes (TP) of analysis.

\section{Numerical modelling and experimental estimation of fibre orientation}

Experimental specimen is injection moulded tensile bar. Used thermoplastic is polypropylene with $20 \%$ volume fraction of glass fibres. Diameter of the fibre is $0.02 \mathrm{~mm}$ and length is about $0.5 \mathrm{~mm}$. Injection moulded temperature is $280^{\circ} \mathrm{C}$, pressure is $6 \mathrm{MPa}$, packing is $3.6 \mathrm{MPa}$ and cooling time is $10 \mathrm{~s}$. In this specimen six places (see Figure 10) were analyzed and each place was examine in each of three directions (X, Y and Z). Fibres close to the mould walls tend to be aligned parallel to the mould fill direction (MFD) (see Figure 9). Examples of microstructure of the analysed place no. 6 in all three cross sections are in Figure 11. Measured and calculated results in two analysed places (1 and 6) are in Table 2.

Table 2. Measured and calculated results in analyzed places 1 and 6 .

\begin{tabular}{ccccccc}
\hline TP & X1 & Y1 & Z1 & X6 & Y6 & Z6 \\
\hline $\mathrm{P}_{\mathrm{i}}$ & 929 & 91 & 196 & 291 & 26 & 383 \\
$\mathrm{P}_{\mathrm{Ai}}$ & 3.2916 & 0.6448 & 0.2777 & 2.0621 & 0.1842 & 0.5428 \\
$\mathrm{a}_{\mathrm{ii}}$ & 0.7810 & 0.1530 & 0.0659 & 0.7393 & 0.0660 & 0.1946 \\
\hline
\end{tabular}




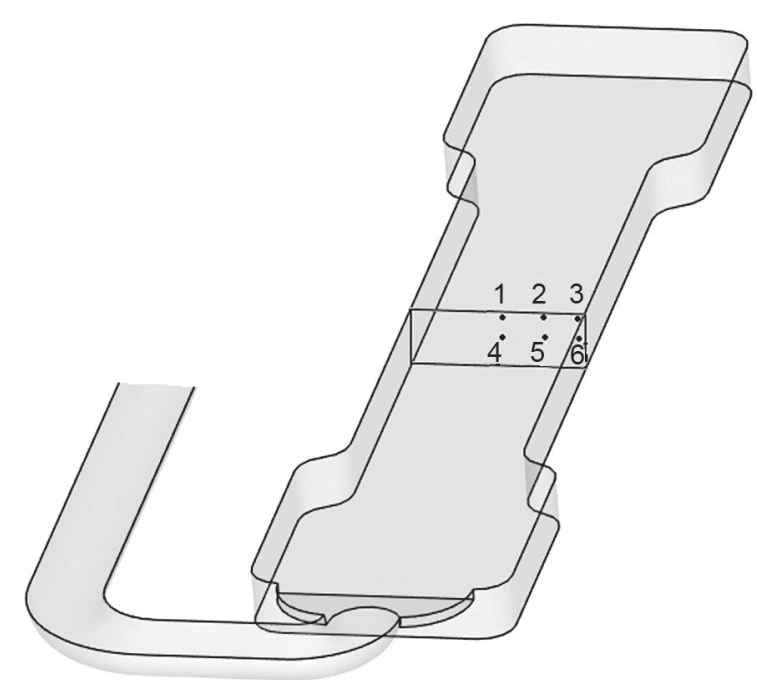

Figure 10. Place of analysis.

Numerical modelling of fibre orientation was realised using Moldex3D software at the same technology conditions. This software allows to view results of fibre orientation as an orientation of the $\mathrm{X}$ direction, $\mathrm{Y}$ direction and $\mathrm{Z}$ direction, which are components of orientation tensor.

Orientation tensors obtained from stereology metallography and numerical simulation are very similar in each from six analysed places. Results obtained from stereology and numerical simulation in 6 analysed places (see Figure 10) are:

Stereology metallography estimated

\begin{tabular}{|c|c|c|c|c|c|c|c|c|}
\hline 0.7810 & 0 & 0 & $(0.7610$ & 0 & 0 & 0.6398 & 0 & 0 \\
\hline 0 & 0.1530 & 0 & 0 & 0.1723 & 0 & 0 & 0.1931 & 0 \\
\hline $1(0$ & 0 & $0.0659)$ & $2(\quad 0$ & 0 & 0.0666 & 0 & 0 & 0.1670 \\
\hline 0.5392 & 0 & 0 & 0.5780 & 0 & 0 & 0.7393 & 0 & 0 \\
\hline 0 & 0.2486 & 0 & 0 & 0.1886 & 0 & 0 & 0.0660 & 0 \\
\hline 4( & 0 & 0.1936 & 5 & 0 & 0.2332 & 6( & 0 & 0.1946 \\
\hline
\end{tabular}

Numerically modelled results

\begin{tabular}{|c|c|c|c|c|c|c|c|c|}
\hline 0.7860 & 0 & 0 & 0.7729 & 0 & 0 & 0.7924 & 0 & 0 \\
\hline 0 & 0.1481 & 0 & 0 & 0.1585 & 0 & 0 & 0.1066 & 0 \\
\hline $1(0$ & 0 & 0.0659 & 2( & 0 & 0.0686 & 0 & 0 & 0.1010 \\
\hline$(0.5701$ & 0 & 0 & $(0.6359$ & 0 & 0 & 0.7751 & 0 & 0 \\
\hline 0 & 0.2363 & 0 & 0 & 0.1648 & 0 & 0 & 0.0767 & 0 \\
\hline 0 & 0 & 0.1936 & 5( & 0 & 0.1993 & 6( & 0 & 0.1482 \\
\hline
\end{tabular}

\section{RESULTS AND DISCUSSION}

The utilization of stereological metallography allows describing structure anisotropy. In case of plastic deformed material, degree of orientation of grain boundaries is determined. Grain boundaries are decomposed into isotropic, planar and linear oriented components - specific surface area of grain boundaries. Stereology method of oriented test lines was used. Consequential conversion of its values to strain leads to determination of deformations in three main axes. Proposed method of conversion of grain boundary degree orientation to grain deformation is independent on the initial grain size in case of idealized tetrakaidecahedron grains shape - strain also depends only on shape of grain and it does not depend on grain dimensions. It allows estimation of local plastic deformation from estimation of microstructure anisotropy in arbitrary place of body with arbitrary state of initial deformation as it was seen in example of analysis drawing process.

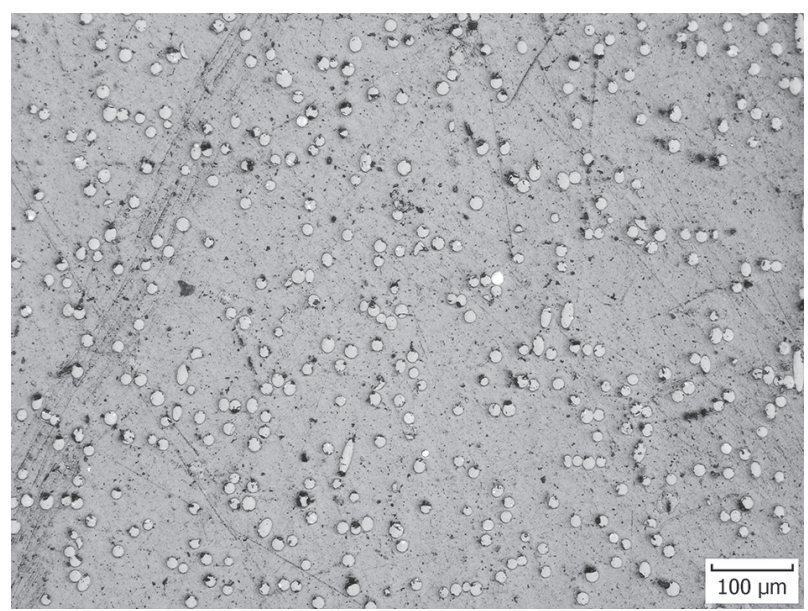

a)

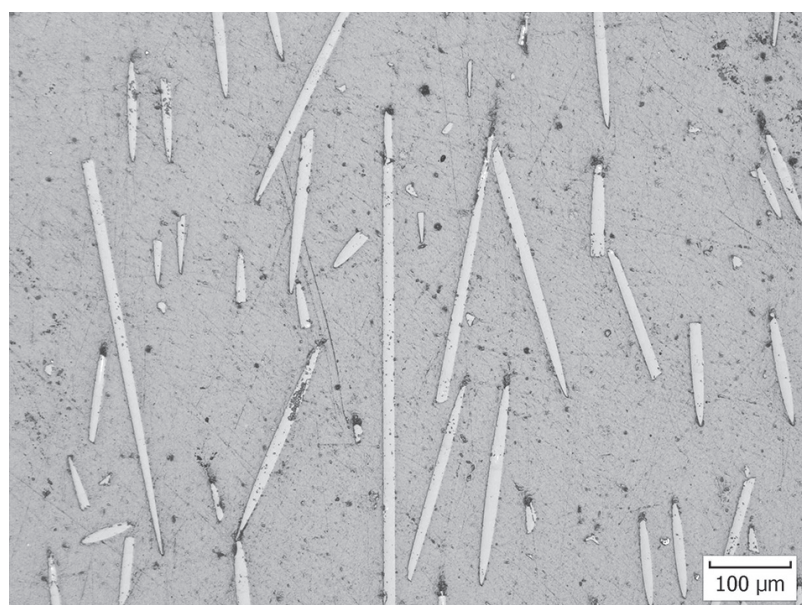

b)

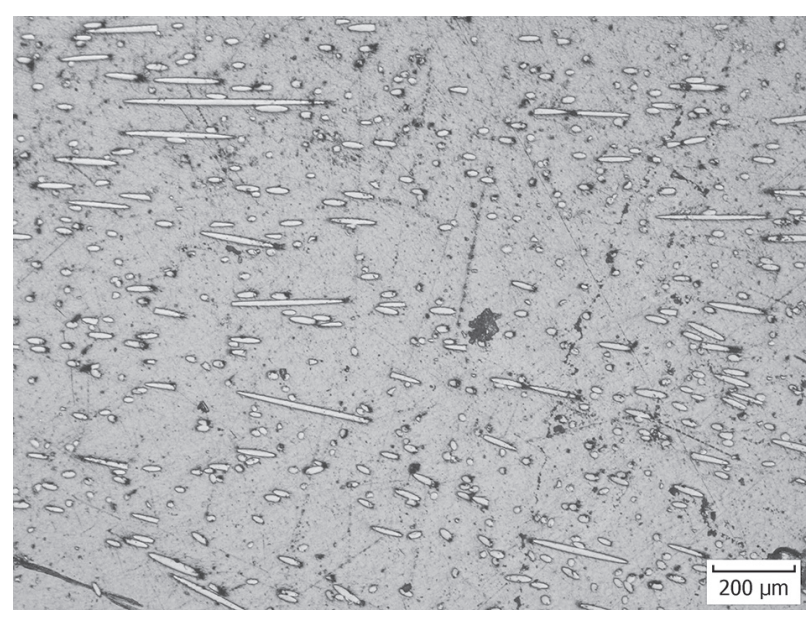

c)

Figure 11. Microstructure of the analyzed place no. 6 in three cross sections; a) $\mathrm{X}, \mathrm{b}$ ) $\mathrm{Y}$, c) Z). 
In case of injection moulding parts, oriented test plane stereology method was used for estimation of experimental orientation of fibres. Degree of orientation is estimated from the number of intersections of fibres with test planes and from these results components of an orientation tensor. This method is very simple in comparison with a current method which is based on measurement of dimensions and orientation of lot of fibre intersections in analysed plane and there are no limits in dimensions or shape of fibres. The fibre orientation can be controlled by injection parameters and these processes are often numerically modelled. Estimation of short glass fibre orientation leads to experimental verification of numerical simulation model, as it was seen in example of analysis injection moulding process. Also results of local plastic deformation estimated from grain boundaries orientation can be used for verification of forming numerical model by comparing these results with numeric simulated ones.

\section{CONCLUSIONS}

The utilization of stereology metallography allows very simple and effective experimental estimation of structure anisotropy of materials. Estimation of grain boundary orientation allows determination of dependence of the degree of orientation to strain. It provides the possibility of setting three main strains in each place in volume of the deformed polycrystalline material. Estimation of short glass fibre orientation allows determination of orientation tensor. It leads to experimental verification of numerical simulation model, which can be optimized to obtain coincidence with experiments. These methods can be applied for all types of materials as metals, plastics or ceramics. In case of ceramics, analysis of deformation of sintered particles or analysis of pores can be done, moreover orientation of ceramic fibres in matrix or fibre orientation in ceramic matrix of composites can be estimated.
Acknowledgement

This work was supported by the VEGA Grant No. 1/0122/16 of the Grant Agency of the Slovak Republic Ministry of Education and by the Slovak Research and Development Agency under the contract No. APVV-150319. Many thanks belong to Lukas Likavcan MSc. for numerical modeling.

\section{REFERENCES}

1. Saltykov S.A. (1970). Stereometric metallography. $3^{\text {rd }}$ ed. Metallurgia.

2. Rios P.R., Glicksmanb M.E. (2006): Modeling polycrystals with regular polyhedra. Materials Research, 9, 231-236. doi:10.1590/S1516-14392006000200021

3. Zhu Q., Sellars M.C., Bhadeshia H.K. D.H. (2007): Quantitative metallography of deformed grains. Materials Science and Technology, 23, 757-766. doi:10.1179/ 174328407 X157308

4. Eberhardt C., Clarke A. (2001): Fibre orientation measurements in short glass fibre composites. Composites Science and Technology, 61, 1389-1400. doi:10.1016/S0266-3538 (01)00038-0

5. Martinkovic M., Minarik S. (2016). Short notes on the grains modification by plastic deformation, in: Hubbard D. (Ed.): Plastic Deformation. NOVA Publishers. pp. 1-44.

6. Hartley C.S. (2006): Strain and the quantitative characterization of anisotropic microstructures. Metallurgical and Materials Transactions A: Physical Metallurgy and Materials Science, 37, 3531- 3539. doi:10.1007/s11661006-1048-0

7. Jung S.W., Kim S.Y., Nam H.W., Han K.S. (2001): Measurement of fiber orientation and elastic-modulus analysis in short-fibre-reinforced composites. Composites Science and Technology, 61, 107-116. doi:10.1016/S02663538(00)00200-1 